\title{
Neurogenic Role of the Depolarizing Chloride Gradient Revealed by Global Overexpression of KCC2 from the Onset of Development
}

\author{
Annie Reynolds, ${ }^{1,2,3}$ Edna Brustein, ${ }^{1,2}$ Meijiang Liao, ${ }^{1,2}$ Adriana Mercado, ${ }^{5}$ Elisa Babilonia, ${ }^{5}$ David B. Mount, ${ }^{5,6}$ and \\ Pierre Drapeau ${ }^{1,2,3,4}$ \\ ${ }^{1}$ Department of Pathology and Cell Biology and Le Groupe de Recherche sur le Système Nerveux Central, Université de Montréal, Montréal, Québec, Canada \\ H3T 1J4, ${ }^{2}$ Centre for Research in Neuroscience, Research Institute of the McGill University Health Centre, Montréal, Québec, Canada H3G 1A4, \\ ${ }^{3}$ Department of Biology, McGill University, Montréal, Québec, Canada H3A 1B1, ${ }^{4}$ Department of Neurology and Neurosurgery, McGill University, Montréal, \\ Québec, Canada H3A 2B4, ${ }^{5}$ Renal Division, Brigham and Women's Hospital, Harvard Institutes of Medicine, Boston, Massachusetts 02115, and ${ }^{\circ}$ Division of \\ General Internal Medicine, Veterans Affairs Boston Healthcare System, Harvard Medical School, West Roxbury, Massachusetts 02132
}

GABA- and glycine-induced depolarization is thought to provide important developmental signals, but the role of the underlying chloride gradient has not been examined from the onset of development. We therefore overexpressed globally the potassium-chloride cotransporter 2 (KCC2) in newly fertilized zebrafish embryos to reverse the chloride gradient. This rendered glycine hyperpolarizing in all neurons, tested at the time that motor behaviors (but not native KCC2) first appear. KCC2 overexpression resulted in fewer mature spontaneously active spinal neurons, more immature silent neurons, and disrupted motor activity. We observed fewer motoneurons and interneurons, a reduction in the elaboration of axonal tracts, and smaller brains and spinal cords. However, we observed no increased apoptosis and a normal complement of sensory neurons, glia, and progenitors. These results suggest that chloride-mediated excitation plays a crucial role in promoting neurogenesis from the earliest stages of embryonic development.

Key words: differentiation; axonal growth; zebrafish; cotransporter; neuronal excitability; glycine

\section{Introduction}

In contrast to most adult neurons, nascent neurons have an elevated intracellular chloride concentration. This change in chloride homeostasis occurs because initially most immature neurons express the sodium-potassium-chloride cotransporter 1 (NKCC1) responsible for chloride uptake but not the neuronspecific, extrusive potassium-chloride cotransporter 2 (KCC2) (Payne et al., 2003). Thus, activation of chloride conducting ion channels gated by GABA or glycine results in depolarization, a phenomenon thought to influence many aspects of embryonic neurodevelopment (Ben-Ari, 2002; Fiumelli and Woodin, 2007). However, this remains to be tested in vivo from the onset of development in all neurons of the CNS.

KCC2 expression is developmentally regulated and often coincides with neuronal maturation and the switch from excitatory to inhibitory actions of GABA and glycine (Rivera et al., 1999;

Received Aug. 20, 2007; revised Nov. 30, 2007; accepted Dec. 29, 2007.

This work was supported by the Veterans Administration and by the National Institutes of Health (D.B.M.), by the Canadian Institute of Health Research and Le Groupe de Recherche sur le Système Nerveux (entral of the Université de Montréal (P.D.), and by the McGill University Health Center Research Institute (A.R.). We are grateful to G. Laliberté and M. Drits for excellent animal care, to J. R. McDearmid, L. Saint-Amant, and N. Champagne for helpful comments on this manuscript, and to R. J. Dunn and M. Lachance for their technical help.

Correspondence should be addressed to Pierre Drapeau, Department of Pathology and Cell Biology, Université de Montréal, Pavillon Roger-Gaudry N-535, 2900, Boulevard Édouard-Montpetit, Montréal, Québec, Canada H3T 1J4. E-mail: p.drapeau@umontreal.ca.

DOI:10.1523/JNEUROSCI.3791-07.2008

Copyright $\odot 2008$ Society for Neuroscience $\quad$ 0270-6474/08/281588-10\$15.00/0
Yamada et al., 2004). KCC2 expression underpins the incorporation of inhibitory neurotransmission into maturing neural networks (Ben-Ari, 2002). Until KCC2 upregulation, chloride channels provide much of the depolarizing activity at times when glutamatergic transmission is primarily silent (Liao and Malinow, 1996). Chloride-mediated depolarization activates voltagedependent calcium channels, thereby periodically elevating cellular calcium concentrations and initiating a range of developmental programs (Ben-Ari, 2002). The trophic depolarizing actions of GABA and glycine have been implicated in proliferation, migration, differentiation, axon pathfinding, dendritic arborization, and synaptogenesis in both immature (Owens and Kriegstein, 2002; Spitzer, 2006) and adult (Ge et al., 2007) nervous systems. Depending on the developmental window studied, interfering with GABAergic or glycinergic transmission affects these processes. However, it remains unclear whether chloridemediated depolarization is the common mechanism underlying the early actions of GABA and glycine. Precocious expression of KCC2 in subsets of neurons (Chudotvorova et al., 2005; Lee et al., 2005; Akerman and Cline, 2006; Liu et al., 2006; Cancedda et al., 2007) can alter dendritic growth and increase or decrease synaptogenesis. The effects depend on the type of preparation or age of the animal, be it cultured neurons or brain slices, from older or younger animals or developing embryos used in in vivo studies. In particular, the effects of reversing the neuronal chloride gradient globally from the onset of in vivo development have yet to be studied in any preparation. 
Recently, we assessed the effects of perturbing glycinergic signaling by antisense knockdown of the embryonic $\alpha 2$ glycine receptor subunit $(\mathrm{GlyR} \alpha 2)$ in the zebrafish embryo (McDearmid et al., 2006). This caused a loss of spinal interneurons and an increase in proliferation, indicating that glycine signaling is required for progenitors to exit the cell cycle and differentiate into interneurons. To determine whether these effects depended on the depolarizing chloride gradient and to examine the importance of the delay in KCC2 expression in this process, we precociously overexpressed KCC2 in the zebrafish blastula, which allowed us to reverse the chloride gradient throughout the embryo from the onset of development. We examined the developmental consequences for the CNS at behavioral, electrophysiological, and cellular levels. Embryonic development was severely compromised as neurogenesis and motor behavior were perturbed. The maintenance of a depolarizing chloride gradient, through delayed expression of KCC2, thus appears to be essential for promoting neuronal differentiation at the earliest stages of development.

\section{Materials and Methods}

Animals. Zebrafish (Danio rerio) embryos were obtained from a breeding colony maintained according to previously established procedures (Westerfield, 1995). All experiments were performed in compliance with the guidelines of the Canadian Council for Animal Care. We used embryos from transgenic lines in which enhanced green fluorescent protein (EGFP) expression is driven by the Neurogenin1 (NGN1) promoter for sensory neuron visualization (Blader et al., 2003) or driven by the proteolipid protein $(P L P)$ promoter for oligodendrocyte progenitor assessment (Yoshida and Macklin, 2005).

Reverse transcription-PCR. The zebrafish $z f k c c 2$ (GenBank accession number XM_001337764) and zfnkccl (GenBank accession number NM_001002080) orthologs were identified (National Center for Biotechnology Information) and used to design specific primers. The $z f k c c 2$ primer sequences were GAGATGCAACATCCAAGGAAAG and CCCGCGTAGATAGCCAGGATGG, and the $z f n k c c 1$ primer sequences were GGTTTTGCCGAAACTGTTGT and AAAAGCGTTCCTTTTGGGAT. Total RNA from zebrafish of different developmental stages was extracted using Trizol (Invitrogen, Carlsbad, CA), and the cDNAs were synthesized with Superscript II Reverse Transcriptase (Invitrogen). PCR was performed using Taq (Fermentas, Burlington, Ontario, Canada). Note that the human $\mathrm{KCC} 2$ gene was used in the overexpression experiments described below.

Xenopus and zebrafish expression constructs. The wild-type human KCC2 gene in the Xenopus expression vector pGEMHE (Song et al., 2002) was mutated at cysteine 568 using the QuikChange mutagenesis system (Stratagene, La Jolla, CA). This cysteine to alanine substitution (C568A) impaired the chloride-extruding function of this cotransporter as measured by rubidium $(\mathrm{Rb})$ uptake (see below).

In some experiments, KCC2 was N-terminal fusion tagged with EGFP to enable visualization of mRNA translation with fluorescence microscopy. PCR was performed with Pfu (Fermentas) and Taq (Expand Long Template; Roche, Indianapolis, IN) to insert an EcoRI site downstream of the start codon and a SalI site downstream of the 31 adenosines poly(A) tail. The primer sequences were TTGAATTCACTCAACAACCTGACGGACTGC and GCGTCGACGCTTGCATGCCTTGCGGCCGC. The PCR product was inserted in a pCRII TOPO vector (Invitrogen), was digested with EcoRI and SalI, and was ligated in pEGFP-C1 using the T4 ligase (New England Biolabs, Ipswich, MA). The EGFP-KCC2 was then digested with SnaBI and SalI and was ligated in a pCS2+ zebrafish expression vector (kindly provided by D. Turner, University of Michigan, Ann Arbor, MI) digested with StuI and XhoI. The construct was then digested with ClaI and NheI to delete a sequence upstream of the EGFP start codon, was treated with Klenow fragments, and was selfcircularized with T4.

Before rubidium uptake was assessed in Xenopus oocytes, subcloning of EGFP-KCC2 into pGEMHE was undertaken to optimize expression, using BamHI and $\mathrm{XbaI}$ after inactivation of an internal BamHI site within the open reading frame (QuikChange; Stratagene). An NheI site within the $5^{\prime}$-flanking multiple-cloning site was subsequently inactivated by mutagenesis, followed by sequencing of the entire EGFP-KCC2 open reading frame.

${ }^{86}$ Rubidium ${ }^{+}$Uptake Assay in Xenopus Oocytes. To express KCC2, EGFP-KCC2, and KCC2-C568A in Xenopus oocytes, the cDNAs inserted in pGEMHE were linearized at the $3^{\prime}$ end using NheI. cRNA was in vitro transcribed using the T7 polymerase mMESSAGE mMACHINE kit (Ambion, Austin, TX). Stage V-VI oocytes were defolliculated and injected with $50 \mathrm{nl}$ of water or with water containing $0.5 \mu \mathrm{g} / \mu \mathrm{l}$ cRNA. Oocytes were incubated at $16^{\circ} \mathrm{C}$ in ND96 medium (in mм: $96 \mathrm{NaCl}, 1.8$ $\mathrm{CaCl}_{2}, 2 \mathrm{KCl}, 1 \mathrm{MgCl}$, and $5 \mathrm{HEPES} /$ Tris, $\mathrm{pH} 7.4$ ) supplemented with 2.5 $\mathrm{mm}$ sodium pyruvate and $5 \mathrm{mg} / 100 \mathrm{ml}$ gentamicin. The uptake was assessed at room temperature $4-5 \mathrm{~d}$ after injection under isotonic conditions: a 30 min incubation period in an $\mathrm{Na}^{+}$and $\mathrm{Cl}^{-}$-free medium [in mM: $50 \mathrm{~N}$-methyl-D-glucamine (NMDG) gluconate, $10 \mathrm{~K}^{+}$-gluconate, $4.6 \mathrm{Ca}^{2+}$-gluconate, $1 \mathrm{Mg}^{2+}$-gluconate, and 5 HEPES/Tris, pH 7.4) containing ouabain $(1 \mathrm{mM})$ followed by a $60 \mathrm{~min}$ uptake period in $\mathrm{a} \mathrm{Na}^{+}$-free medium (in mM: $50 \mathrm{NMDG}-\mathrm{Cl}, 10 \mathrm{KCl}, 1.8 \mathrm{CaCl}_{2}, 1 \mathrm{MgCl}_{2}$, and 5 HEPES/Tris pH 7.4) supplemented with ouabain and $2.5 \mu \mathrm{Ci}$ of ${ }^{86} \mathrm{Rb}^{+}$ per milliliter (PerkinElmer, Wellesley, MA)]. Isotonic conditions were generated by supplementation with $3.5 \mathrm{~g} / 100 \mathrm{ml}$ sucrose to reach isomolar conditions for oocytes $(\sim 210 \mathrm{mOsm} / \mathrm{kg})$. Ouabain was added to inhibit ${ }^{86} \mathrm{Rb}^{+}$uptake via the $\mathrm{Na}^{+}-\mathrm{K}^{+}-$ATPase. Blockade of $\mathrm{Na}^{+}$extrusion with this method prevented ${ }^{86} \mathrm{Rb}^{+}$uptake via the endogenous oocyte $\mathrm{Na}^{+}-\mathrm{K}^{+}-2 \mathrm{Cl}^{-}$cotransporter. After ${ }^{86} \mathrm{Rb}^{+}$uptake, oocytes were washed three times in ice-cold uptake solution without isotope to remove extracellular fluid tracer. Oocytes were dissolved in 10\% SDS, and tracer activity was determined for each oocyte by $\beta$-scintillation counting. A minimum of 15 oocytes were used for each experimental condition.

KCC2 overexpression in zebrafish embryos. KCC2 mRNAs were transcribed from KpnI-linearized pCS2 + constructs using the SP6 polymerase mMESSAGE mMACHINE kit (Ambion). The mRNAs were diluted in nuclease-free water (Ambion), and the vital dye fast green $(0.05 \%$; Sigma, St. Louis, MO) was added to enable confirmation of injection. KCC2 mRNA ( $100-150 \mathrm{ng} / \mu \mathrm{l}$ in a $2-3 \mathrm{nl}$ volume) was injected into oneto four-cell stage blastulae using a Picospritzer III (General Valve, Fairfield, NJ) pressure ejector. Protein translation was confirmed via visualization of EGFP fluorescence with a compound epifluorescent microscope (Nikon, Tokyo, Japan). Morphology and behavioral touch responses were assessed with a stereomicroscope (Zeiss, Oberkochen, Germany). To observe the swimming behavior, the embryos were anesthetized in $0.02 \%$ tricaine (Sigma) and embedded in $2 \%$ agarose (Invitrogen). After solidification, the agarose was cut and removed so as to freely expose the tail but by keeping the head of the embryos firmly immobilized. Tricaine was washed out, and response to touch was recorded using a Photron (San Diego, CA) Fastcam PCI high-speed (500 frames/s) video camera.

Gramicidin-perforated-patch recordings. The chloride reversal potential of spontaneously active spinal neurons was determined in control embryos and embryos overexpressing KCC2 or KCC2-C568A. The amplitude of subthreshold spontaneous synaptic glycinergic activity was determined at different holding currents using non-invasive gramicidinperforated-patch recording to preserve the intracellular chloride concentration. To do this, intact embryos were paralyzed with $0.01-0.02 \%$ mivacurium chloride (Mivacron; GlaxoSmithKline, Brentford, UK) and embedded in 2\% low-melting-point agarose (Invitrogen). The agarose was immersed in Evans solution [in mM: $134 \mathrm{NaCl}, 2.9 \mathrm{KCl}, 2.1 \mathrm{CaCl}_{2}, 1.2$ $\mathrm{MgCl}_{2}, 10$ HEPES, and 10 glucose, pH 7.8 (290 mOsm)]. The tip of the patch pipette $(\sim 8 \mathrm{M} \Omega)$ was first dipped into a gramicidin-free solution [in mM: $132 \mathrm{KCl}, 2 \mathrm{MgCl}_{2}, 10 \mathrm{HEPES}, 10$ EGTA, and $4 \mathrm{Na} 2 \mathrm{ATP}(290$ mOsm), $\mathrm{pH}$ 7.2]; then the pipette was backfilled with the same solution that contained $10 \mu \mathrm{g} / \mathrm{ml}$ gramicidin (from a $10 \mathrm{mg} / \mathrm{ml}$ stock solution in DMSO). Sulforhodamine B (2\%) was added to the pipette solution to permit visualization of membrane rupture at the end of each experiment with fluorescence microscopy. Spinal neurons were visualized using Hoffman modulation optics ( $40 \times$ water immersion objective) and re- 
cordings started after the input resistance had stabilized (150-350 M $\Omega$ ). Data were recorded with an Axopatch 200B amplifier and digitized online at $40 \mathrm{kHz}$ using Clampex 8.0 software (Molecular Devices, Union City, CA). At the end of some experiments, spontaneous glycinergic synaptic bursts were blocked using strychnine ( $1 \mu \mathrm{M}$; Sigma) to confirm their glycinergic nature.

In an additional experimental paradigm, we verified KCC2 activity in silent nonspiking immature neurons by comparing their resting potential before and after bath administration of stock solutions: bumetanide (100 $\mu \mathrm{M})$, furosemide (1-5 mM), and $R(+)$-butylindazone (DIOA) (1 $\mathrm{mm}$ ) (all from Sigma). Considering that the embryos were intact and embedded in agarose, we assume that the final drug concentrations were 10 times lower (Brustein et al., 2003; Brustein and Drapeau, 2005).

Whole-mount immunohistochemistry. Embryos were dechorionated and anesthetized in $0.02 \%$ tricaine (Sigma) and subsequently fixed in $4 \%$ paraformaldehyde dissolved in phosphate buffer for $2 \mathrm{~h}$ at room temperature or overnight at $4^{\circ} \mathrm{C}$. After extensive washing, the embryos were permeabilized for $30 \mathrm{~min}$ with distilled water and incubated $1 \mathrm{~h}$ in blocking solution ( $2 \%$ goat serum, $1 \%$ bovine serum albumin, $1 \%$ dimethylsulfoxide, and $0.1 \%$ Triton X-100 in PBS) and then overnight at room temperature in primary antibody diluted in blocking solution. Primary antibodies used were anti-Hu (1:50; Invitrogen), anti-acetylated tubulin (1:100; Sigma), anti-neurofilament-associated antigen [3A10; 1:25; obtained from the Developmental Studies Hybridoma Bank (DSHB), University of Iowa, Iowa City, IA], anti-HB9 (1:20; DSHB), anti-Zrf-1 (1:50; DSHB), anti-Pax2 (1:100; Covance, Princeton, NJ), anti-Dbx1 (1:75; kindly provided by T. M. Jessell, Columbia University, New York, NY), anti-phospho-histone-H3 (1:300; Upstate, Waltham, MA), and antiGFP (1:100; Invitrogen). Embryos were washed with PBTx (0.1\% Triton $\mathrm{X}-100$ in PBS) and incubated overnight at room temperature in Alexa 488- or Alexa 568-conjugated secondary antibodies diluted in blocking solution (1:750; Invitrogen). After rinsing in PBTx, embryos were incubated in $50 \%$ glycerol in PBS and mounted on coverslips. Imaging was performed using an Ultraview LCI confocal microscope with MetaMorph imaging software (PerkinElmer, Woodbridge, Ontario, Canada).

Neurodegeneration and bromodeoxyuridine assays. To stain degenerating cells, the vital dye acridine orange ( $5 \mu \mathrm{g} / \mathrm{ml}$; Sigma) was injected in the yolk of dechorionated embryos. After $1.5 \mathrm{~h}$, the live embryos were mounted on a coverslip, and apoptotic cells were visualized using an epifluorescence microscope (Nikon).

To stain proliferating cells in the S-phase of the cell cycle, embryos were dechorionated, anesthetized in tricaine, and injected in the yolk with $20 \mathrm{~mm}$ bromodeoxyuridine (BrdU). After $40 \mathrm{~min}$, the embryos were fixed in $4 \%$ paraformaldehyde for $3 \mathrm{~h}$. After extensive washing, the embryos were rinsed twice with methanol and incubated in fresh methanol at $-20^{\circ} \mathrm{C}$ for $1 \mathrm{~h}$ or overnight. Rehydration was performed using graded methanol/PBS series. The embryos were then washed first with PBTx and then with water. After two rinses and $1 \mathrm{~h}$ incubation in $2 \mathrm{~N} \mathrm{HCl}$ and after several washes in PBTx, the embryos were placed in blocking solution. The remainder of the immunohistochemistry procedure described above was performed, using the mouse monoclonal anti-BrdU antibody (1:50; Sigma) to detect proliferating cells that had incorporated BrdU.

Statistics. The phenotypic traits used to quantify the effects of KCC2 overexpression on embryonic morphology and on tubulin-marked brain and spinal cord structures were compared using $\chi^{2}$ testing. Using $2 \mu \mathrm{m}$ $z$-series stacks obtained from the MetaMorph imaging software, the labeled cells in a $100 \mu \mathrm{m}$ longitudinal section of the spinal cord located at the level of the anal somite were counted. Significance across treatments was compared using a Student's $t$ test. Data are represented as mean \pm SEM.

\section{Results}

\section{Developmental expression of KCC2 in zebrafish}

Before attempting to overexpress KCC2, we determined the temporal expression pattern of the endogenous zebrafish $z f k c c 2$ and compared it with $z f n k c c 1$, which is expressed throughout development and adulthood in other species (Plotkin et al., 1997). Because to date neither gene has been cloned from zebrafish, the
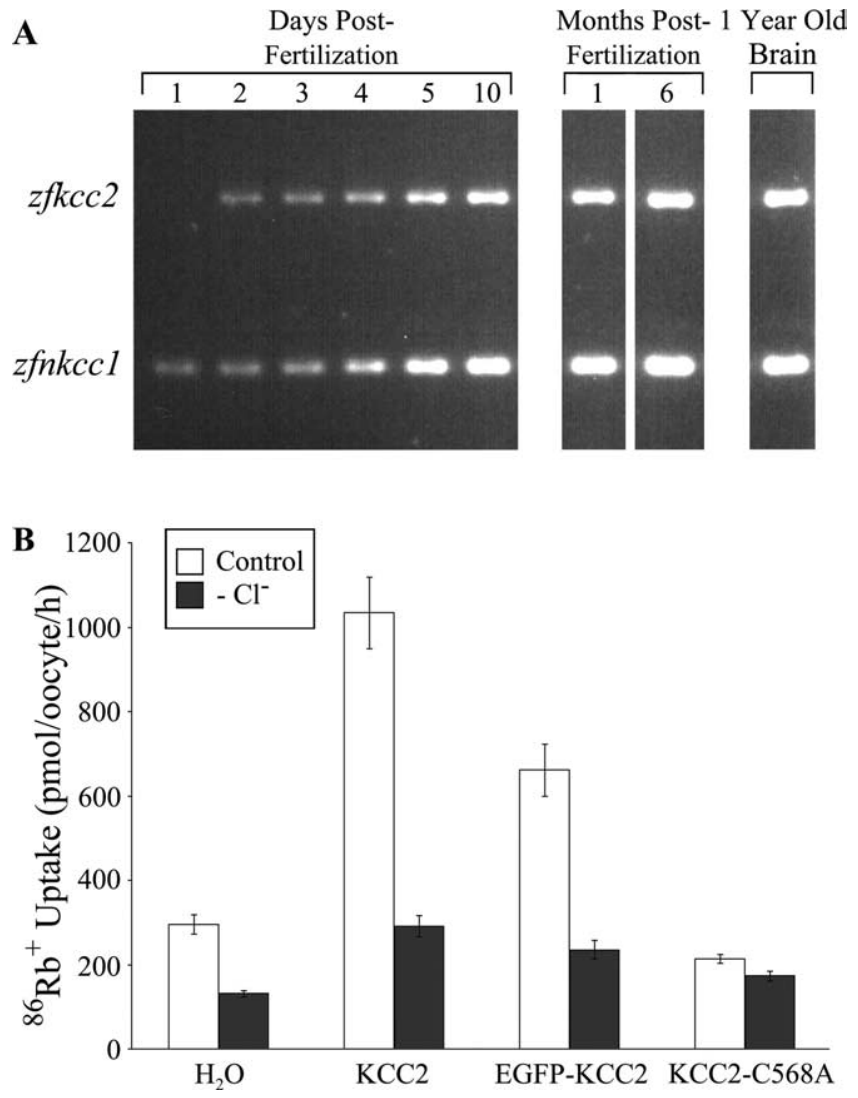

Figure 1. Zebrafish zfkcc2 expression is absent in embryonic neurons. A, RT-PCR using zebrafish total RNA from animals aged $1-5 \mathrm{dpf}, 10 \mathrm{dpf}$, and 1 and 6 months postfertilization, and 1 year old brain tissue. The top band represents zfkcc 2 mRNA (778 bp), and the bottom band corresponds to the zfnkcc $1 \mathrm{mRNA}$ (413 bp). zfkcc2 is initially absent (at $1 \mathrm{dpf}$ ) and appears at 2 $\mathrm{dpf}$, whereas zfnkcc $1 \mathrm{mRNA}$ is detectable at all ages tested. $\boldsymbol{B}$, Activity of the wild-type $K C(2$, the EGFP-tagged $K C(2$, and the C568A mutation. Xenopus 0ocytes were transfected with cRNA from the three isoforms or with water. Activity of the cotransporters was assessed in isotonic conditions, in both the presence (Control) and absence $\left(-\mathrm{Cl}^{-}\right)$of chloride, by measuring oocyte uptake of ${ }^{86}$ rubidium ${ }^{+}$.

orthologs were identified via basic local alignment search tool searches of the zebrafish genome using conserved mammalian KCC2 and NKCC1 DNA and protein sequences. The predicted $z f k c c 2$ and $z f n k c c 1$ were, respectively, 71.4 and $67.5 \%$ identical at the nucleotide level and 74.3 and $75.0 \%$ identical at the amino acid level to human sequences. Moreover, the predicted transmembrane segments and the large intracellular $\mathrm{C}$ termini shared 75-85\% identity when comparing human and zebrafish proteins, allowing us to unambiguously identify the $z f n k c c 1$ and zfkcc2 orthologs.

Using reverse transcription (RT)-PCR, we confirmed the presence of $z f n k c c l$ at all developmental stages examined. In contrast, $z f k c c 2$ was first detected at approximately the time of hatching $(2 \mathrm{~d})$ (Fig. 1A). Therefore, the effects of KCC2 overexpression on neurodevelopment were assessed between 26 and $32 \mathrm{~h}$ postfertilization (hpf), before onset of endogenous expression.

\section{Ion transport activity of $\mathrm{KCC} 2$ isoforms}

Because $z f k c c 2$ has not been molecularly cloned, we chose to use the human KCC2 gene as a tool to perform the overexpression experiments in zebrafish. This seemed feasible for our purposes as expression of human and rat KCC2 orthologs has proven successful in Xenopus oocytes (Song et al., 2002; Akerman and Cline, 2006). To overexpress KCC2 in zebrafish, we used both the wild- 


\section{A. EGFP-KCC2 Spinal neurons}
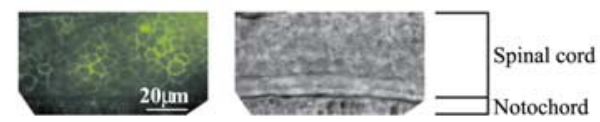

B. Spinal neurons
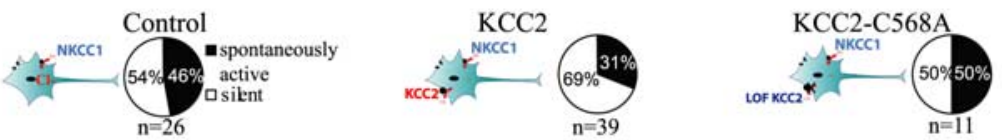

C. Spontaneously active neurons

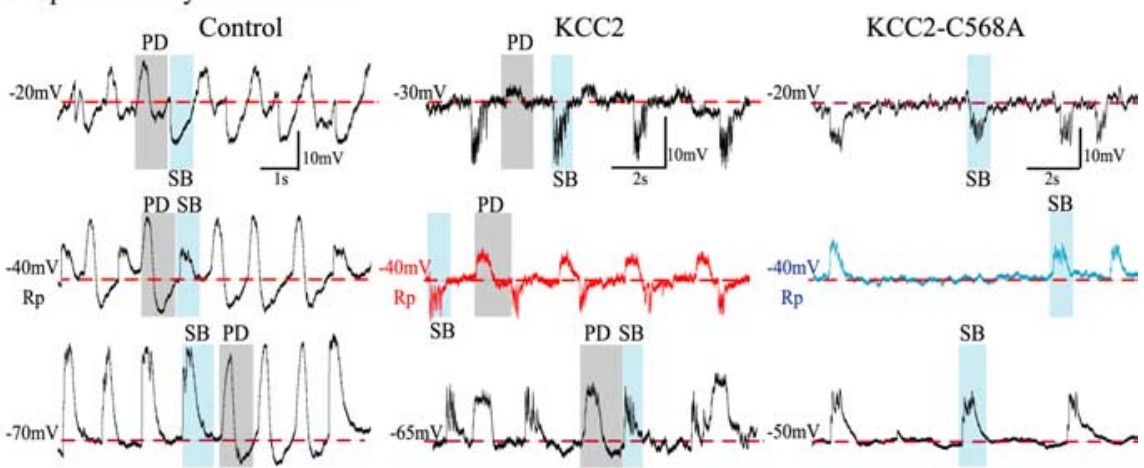

Ci Extended traces of SBs

Cii Reversal potential of SBs

Ciii Average driving force of SBs
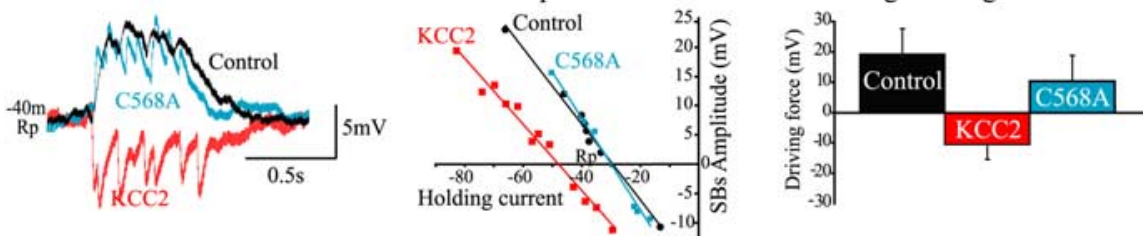

D. Silent neurons
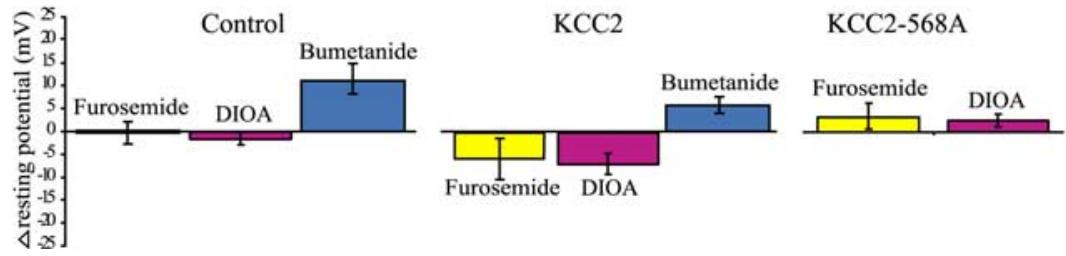

Figure 2. KCC2 overexpression reverses the depolarizing chloride gradient. A, EGFP fluorescence of spinal cord neurons from EGFP-KCC2-overexpressing embryos. Note that the expression is localized to the surface and that all neurons express the cotransporter. $\boldsymbol{B}$, Pie charts illustrating the proportions of silent neurons (white) associated with immaturity and spontaneously active neurons (black) in control, $\mathrm{KCC} 2-$, and $\mathrm{KCC} 2-\mathrm{C} 568 \mathrm{~A}-$ overexpressing embryos. The diagrams illustrate the experimental paradigm. C, Representative traces of spontaneous activity recorded from neurons in control, KCC2-, and KCC2-C568Aoverexpressing embryos. In each case, examples of PDs, which are gap junction mediated, are highlighted by gray and include a large depolarization followed by afterhyperpolarization. Examples of $S B s$, which are chloride mediated, are highlighted in blue. Note that, in neurons from control and KCC2-C568A embryos, SBs are depolarizing from rest $(-40 \mathrm{mV})$, although they are hyperpolarizing from rest in neurons from $\mathrm{KCC} 2$ embryos, as illustrated by extended superimposed SBs traces ( $\boldsymbol{C}$, taken from shaded examples in $C,-40 \mathrm{mV}$ traces). Cii, The reversal potential of SBs (taken from the examples in $C$ ) in control (black circles) and $\mathrm{KCC}-\mathrm{C} 568 \mathrm{~A}$ neurons (blue squares) was approximately $-30 \mathrm{mV}$ [above resting potential (Rp)], whereas that of the $\mathrm{KCC}$-overexpressing neuron (red squares) was more negative $(-47 \mathrm{mV}$; below resting potential). Ciii, The average driving force calculated for each group is positive for control and KCC2-C568A-overexpressing embryos, whereas that of KCC2 embryos is negative, suggesting a reversal of the depolarizing chloride gradient in KCC2 neurons. D, To verify whether KCC2 cotransporter was functionally expressed in silent neurons, we compared the change in resting potential ( $\Delta$ resting potential) in response to application of bumetanide, a more specific blocker of NKCC 1 cotransporter, with that of furosemide and DIOA, which are more specific blockers of KCC2. In control neurons, as expected in the absence of $\mathrm{KCC}$, furosemide and DIOA did not affect the resting potential, whereas bumetanide shifted it to more positive values (bumetanide control vs bumetanide KCC2, Student's $t$ test, $p=$ $0.2)$. In contrast, in $\mathrm{KCC}$-overexpressing embryos, furosemide and DIOA each caused a negative shift in the resting potential (furosemide or DIOA in control vs furosemide or DIOA, in KCC2-overexpressing embryos, $p<0.05$, Student's $t$ test). In KCC2C568A embryos, such a negative shift was not observed.

type and a mutant C568A isoform we predicted to exhibit impaired chloride extrusion. In addition, in some experiments, KCC2 mRNA was tagged at the $5^{\prime}$ end with a sequence encoding EGFP to permit in vivo visualization of translation. To determine whether the various constructs encoded functional proteins, Xenopus oocytes were injected with cRNA, and cotransporter activity was assessed by quantification of chloride-dependent $\mathrm{Rb}$ transport (Song et al., 2002) (Fig. $1 B$ ). The rate of accumulation of radiolabeled ${ }^{86} \mathrm{Rb}$ uptake was $295 \pm$ $23 \mathrm{pmol} /$ oocyte per hour in water-injected oocytes, $1034 \pm 85 \mathrm{pmol} /$ oocyte per hour in KCC2-injected oocytes, $661 \pm 61 \mathrm{pmol} /$ oocyte per hour in EGFP-KCC2-injected oocytes, and $214 \pm 11 \mathrm{pmol} /$ oocyte hour in oocytes injected with the KCC2-C568A mutant. The rates of uptake were $131 \pm 8$, $292 \pm 25,235 \pm 22$, and $173 \pm 12 \mathrm{pmol} /$ oocyte per hour, respectively, in the absence of chloride. Exogenous KCC2 activity was thus 4.5 -fold and 2.6-fold higher in KCC2-injected and EGFP-KCC2-injected cells $(p<0.00001)$ than in water-injected controls; KCC2-C568A was, however, essentially inactive and was used as a negative control.

\section{KCC2 overexpression reverses the chloride gradient}

Embryos injected with EGFP-KCC2 expressed fluorescence throughout the nervous system, in particular at the surface of neurons (Fig. 2A), indicating proper translation and membrane insertion of the EGFP-tagged cotransporter. To determine whether KCC2 effectively modified the chloride gradient in vivo, we recorded from embryonic neurons using the non-invasive gramicidin-perforated-patch technique because it does not interfere with the intracellular chloride concentration (Kyrozis and Reichling, 1995). Current-clamp mode was used because the high input resistance of embryonic neurons in series with that of the perforated patches, together with gap-junctional coupling of early embryonic neurons (Saint-Amant and Drapeau, 2000), made it impossible to reliably voltage clamp cells.

In control and KCC2-C568A embryos, the proportion of spontaneously active spinal neurons was 46 and 50\%, respectively, whereas the remaining neurons were silent ( $n=26$ control and $n=11$ KCC2-C568A) (Fig. $2 B$ ). Silent neurons, which are typically immature (Saint-Amant and Drapeau, 2000), had a normal resting potential and input resistance but showed no synaptic or electrical activity and no activity in response to current injection, thus indicating that they had not yet developed active voltage-gated conductances. In neurons from embryos overexpressing KCC2 $(n=39)$, the proportion of spontaneously active neurons decreased to $31 \%$, whereas the number of silent neurons increased to $69 \%$.

Two types of spontaneous recurrent activity were reported 
previously in embryonic neurons using whole-cell patch recordings (Saint-Amant and Drapeau, 2000): periodic depolarizations (PDs) and synaptic glycinergic bursts (SBs). Both types of activity could be distinguished in gramicidin-perforated-patch recordings (Fig. 2C). PDs (Fig. 2C, gray shading) consist of large depolarization, often followed by an afterhyperpolarization. Because they are generated by passive current spread through gap junctions, their polarity is independent of the holding current. The spontaneous subthreshold SBs (Fig. 2C, blue shading) are a strychnine-sensitive glycinergic chloride conductance (SaintAmant and Drapeau, 2000). Therefore, we measured the amplitude and polarity of SBs to detect changes in the chloride gradient after overexpression of KCC2. Note that no significant GABAergic activity is observed at this stage of zebrafish development (Triller et al., 1997; Buss and Drapeau, 2001).

In neurons from control embryos $(n=4)$ and embryos expressing KCC2-C568A $(n=3)$, SBs were always depolarizing from rest (Fig. 2C, black and blue traces at resting potential expanded in $\mathrm{Ci}$ ) as would be expected for neurons with a high intracellular chloride content (Saint-Amant and Drapeau, 2000; Brustein and Drapeau, 2005). In contrast, among the spontaneously active neurons from embryos expressing KCC2 $(n=5)$, SBs were either not obvious at rest or were hyperpolarizing from rest (Fig. $2 C$, red trace at resting potential, expended in $C i$ ), indicating a reversal of the chloride gradient. As shown in greater detail in Figure 2Cii for the same experiment as in Figure $2 C$, the reversal potential of SBs was more negative $(-47 \mathrm{mV})$ in KCC2 embryos than in control and KCC2-C568A embryos (-30 mV). Considering that, in control, KCC2-C568A-expressing and KCC2expressing neurons (Saint-Amant and Drapeau, 2000, 2001), the average resting potential (normally low) was similar $(-45 \pm 1$, $-41 \pm 1$, and $-42 \pm 1 \mathrm{mV}$, respectively), the average driving force of SBs (Fig. 2Ciii) was positive in control and KCC2C568A-expressing embryos ( $19 \pm 3$ and $10 \pm 3 \mathrm{mV}$, respectively) whereas it was negative in KCC2-expressing embryos $(-11 \pm 2$ $\mathrm{mV}$ ). The changes in driving force observed were similar in magnitude to what was reported in cultured neurons overexpressing KCC2 (Lee et al., 2005). These results demonstrate that overexpressing KCC2 effectively reversed the chloride gradient in vivo, whereas the KCC2-C568A mutant was ineffective.

Because silent neurons lacked chloride-dependent synaptic innervation, we attempted to determine whether they also expressed functional KCC2 using a pharmacological approach. We compared the effects of bumetanide, a more selective antagonist of NKCC1 at low concentrations, to furosemide and DIOA, both more selective antagonists of KCC2 (Russell, 2000), on the resting potential (final concentrations of 10, 100-500, and $100 \mu \mathrm{M}$, respectively). Gramicidin patches allowed us to follow chloridedependent fluctuations of the resting potential during cotransporter blockade. In neurons from control embryos, as expected in the absence of KCC2, furosemide $(n=6)$ and DIOA $(n=3)$ did not affect the resting potential, whereas bumetanide $(n=3)$ caused a shift of $+11.0 \pm 3.0 \mathrm{mV}$, exposing the constitutive activity of NKCC1 (Fig. 2D). Similarly, bumetanide caused a positive shift in the resting potential of silent neurons overexpressing KCC2 $(n=5 ;+5.2 \pm 1.0 \mathrm{mV})$. However, in these neurons, furosemide $(n=6)$ and DIOA $(n=3)$ caused negative shifts of $-5.8 \pm 4.5$ and $-7.3 \pm 1.5 \mathrm{mV}$, respectively (Fig. $2 \mathrm{D}$ ). This is likely to arise from blockade of KCC2 activity, resulting in acute intracellular accumulation of chloride. The resting potential of neurons in embryos expressing KCC2-C568A did not undergo such a negative shift in response of furosemide $(n=3)$ or DIOA $(n=3)$ (Fig. 2D). These results suggest that KCC2 was functionally expressed in silent neurons.

\section{KCC2 overexpression perturbs development}

KCC2 overexpression resulted in several abnormal morphological features (Fig. 3A). The overall size of embryos expressing KCC2 was reduced. The head and the eyes were smaller, the embryos were pigmentless and the tail curvature was pronounced. Aside from the abnormal morphology, no other physical malformations were obvious. KCC2-expressing embryos also exhibited abnormal locomotor behaviors (Fig. 3B). By 27 hpf, zebrafish embryos normally escape in response to touch by producing alternating contractions (Saint-Amant and Drapeau, 1998). In contrast, embryos overexpressing KCC2 generated slow coils in response to touch. Locomotor activity in embryos expressing KCC2-C568A was indistinguishable from control embryos. To assess the significance of precocious KCC2 overexpression, we compared three phenotypic traits: the presence of a small head, small eyes, and abnormal locomotion (i.e., slow coils instead of swimming) $\chi^{2}$ analysis revealed that embryos injected with KCC2 mRNA were significantly different from control ( $p<$ $0.001)$ and $K C C 2-C 568 A$ injected-embryos $(p<0.001)$ for all three traits, whereas $K C C 2-C 568 A$-injected and control embryos did not significantly differ $(p=0.78$ for the eyes, $p=0.06$ for the head, $p=0.40$ for swimming; $n=102 \mathrm{KCC} 2, n=108 \mathrm{KCC} 2-$ C568A, and $n=95$ control). Thus, overexpression of KCC2 from the onset of development affected the gross morphology of the embryos compared with control embryos and perturbed early swimming behaviors.

\section{KCC2 overexpression impairs neural development in the brain and hindbrain}

We selected KCC2-expressing embryos with an obvious developmental phenotype as described above for more detailed cellular analyses. As a first level of analysis, we used the acetylated- $\alpha$ tubulin marker for stable microtubules to assess overall axonal growth in the brain (Fig. $4 A$ ). In control animals $(n=94)$, the ventral and dorsal longitudinal tracts, the postoptic, anterior, and posterior commissures, the trigeminal ganglion, and the optic cup were readily distinguishable (Wilson et al., 1990). In KCC2 embryos, only the ventral longitudinal tract was visible although faint, none of the commissures were apparent, the trigeminal ganglion was absent, and the optic vesicle had not invaginated to form the optic cup $\left(n=53 ; \chi^{2}\right.$ test, $\left.p<0.001\right)$.

Because the hindbrain also appeared smaller in $\mathrm{KCC} 2 \mathrm{em}-$ bryos, axon tract development was investigated in this structure that is essential for sensorimotor integration of early touch responses. In control embryos $(n=14)$, the large Mauthner cells labeled with a neurofilament marker had adopted their characteristic shape and size and extended their axon caudally (Fig. 4B). In contrast, axons of the Mauthners in KCC2-expressing embryos $(n=4)$ were smaller and not as well defined despite projecting correctly. Similarly, the other reticulospinal neurons of the hindbrain and the large axonal tracts extending in the spinal cord exhibited growth defects in KCC2 embryos as the axon bundles were less elaborated (Fig. 4C). This suggests that neural development and consequent axon maturation in both the brain and hindbrain were disturbed by early KCC2 expression and is consistent with the perturbation of touch-evoked responses in these embryos (Fig. 3B). As described below, these axonal tract deficits were likely a consequence of a dramatic reduction in cell numbers. 
A

\section{Control}

$\mathrm{KCC} 2$

KCC2-C568A
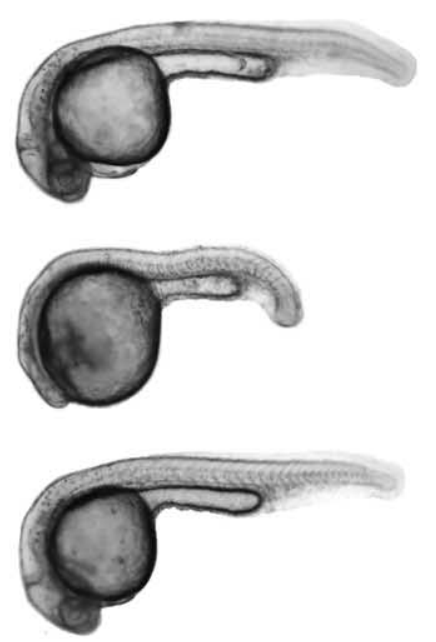

B

Control
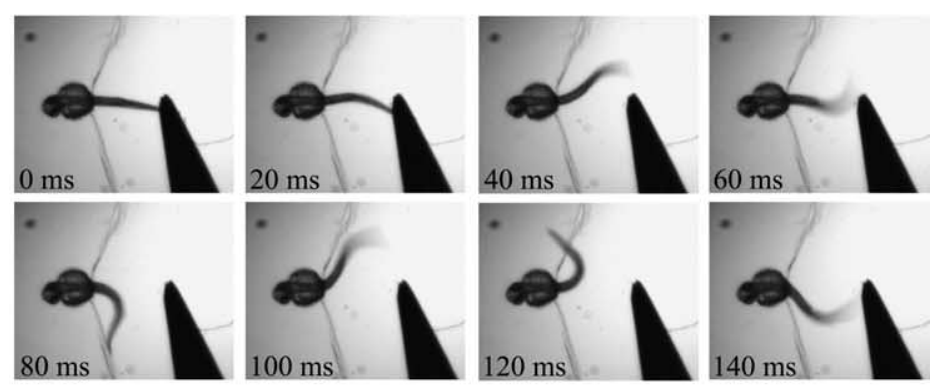

$\mathrm{KCC} 2$
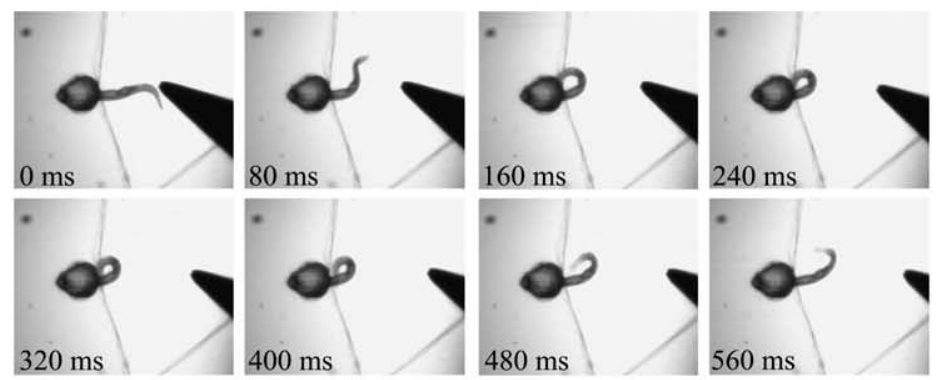

$320 \mathrm{~ms}$
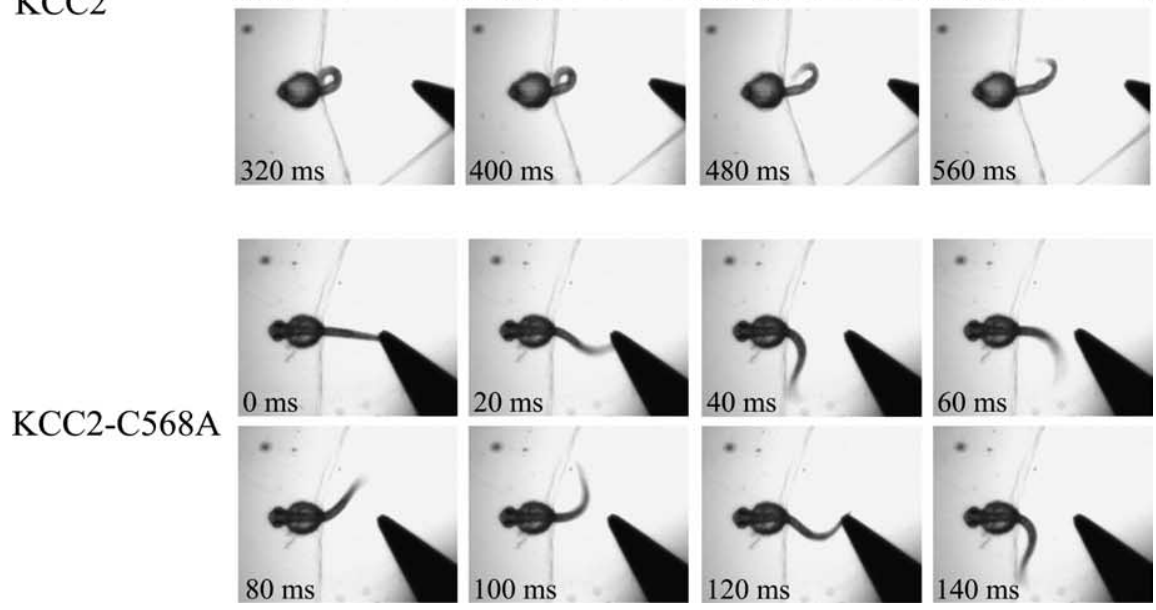

Figure 3. KCC2 overexpression alters the gross morphology and the early swimming behavior of the embryo. $A$, Morphological phenotype of control, KCC2-, and KCC2-C568A-injected embryos. $\boldsymbol{B}$, Response to touch of control, KCC2-, and KCC2-C568Aoverexpressing embryos, whose head was immobilized in agarose. To elicit an escape response, the freely moving tip of the tail was gently touched using a pair of forceps. Note the different timescales in control and KCC2-C568A embryos, which responded with rapid alternating contractions, versus KCC2 embryos, which produced slow coils in response to the stimulus.

tions in the spinal cord. As observed in the brain and hindbrain, precocious expression of KCC2 diminished the elaboration and complexity of spinal axon tracts (Fig. $5 A$ ). Although axons of the caudal primary motoneurons projected ventrally, they often failed to extend completely out of the spinal cord in KCC2 embryos $\left(n=53 ; \chi^{2}\right.$ test, $p<0.001)$.

To determine whether this perturbation in axonal maturation was attributable to disturbances in neurogenesis, we used markers for early developmental events. Newly differentiated neurons, expressing the RNA binding protein $\mathrm{Hu}$ found in neuronal cells leaving the mitotic cycle (Szabo et al., 1991) (Fig. 5B), were significantly less numerous in KCC2-expressing embryos (Table 1). The anti-Pax 2 antibody was used to label subtypes of early differentiating interneurons (Burrill et al., 1997)

(Fig. 5C). Motoneurons were labeled using an antibody against HB9, a homeobox gene necessary for motoneuron differentiation (Arber et al., 1999) (Fig. 5D). Sensory neurons were visualized using a transgenic line of zebrafish expressing EGFP under the control of the NGN1 promoter (Blader et al., 2003) (Fig. 5E). In KCC2-expressing embryos, the population of sensory neurons was unaffected, but only half of the interneurons and motoneurons were observed (Table 1).

We next examined whether the reduction in neurogenesis was attributable to cell death or reduced proliferation. To determine whether the massive loss of neurons was attributable to substantial cell death, apoptotic cells were stained with the vital dye acridine orange (Furutani-Seiki et al., 1996). The spinal cord of control ( $n=$ $5)$ and KCC2-expressing $(n=12)$ embryos had very few apoptotic cells (Fig. 6A). A similar lack of increased apoptosis was observed in the brain and hindbrain (data not shown). These results indicate that a massive increase in cell death did not cause the phenotype, although we cannot rule out neural tract degeneration in the absence of cell death.

To determine whether the selective impairment in neurogenesis resulted from a change in proliferation rather than cell death, as was previously reported after GlyR $\alpha 2$ knockdown (McDearmid et al., 2006), we estimated the number of mitotic progenitor cells using two distinct immunohistochemical methods: BrdU pulse la-

\section{KCC2 overexpression perturbs neuronal differentiation in the spinal cord}

Because of the reduction in trunk size and altered swimming activity as well as the accessibility for more detailed cellular analyses, we closely examined the development of neuronal popula- beling to visualize cells in the S-phase of the cell cycle (Fig. 6B) and phosphorylated-histone- $\mathrm{H} 3$ (PH3) antibody labeling to visualize cells undergoing histone modification during mitosis (Fig. 6C). Using both measures, we found no increase in proliferation during KCC2 overexpression (Table 1). We also labeled a 


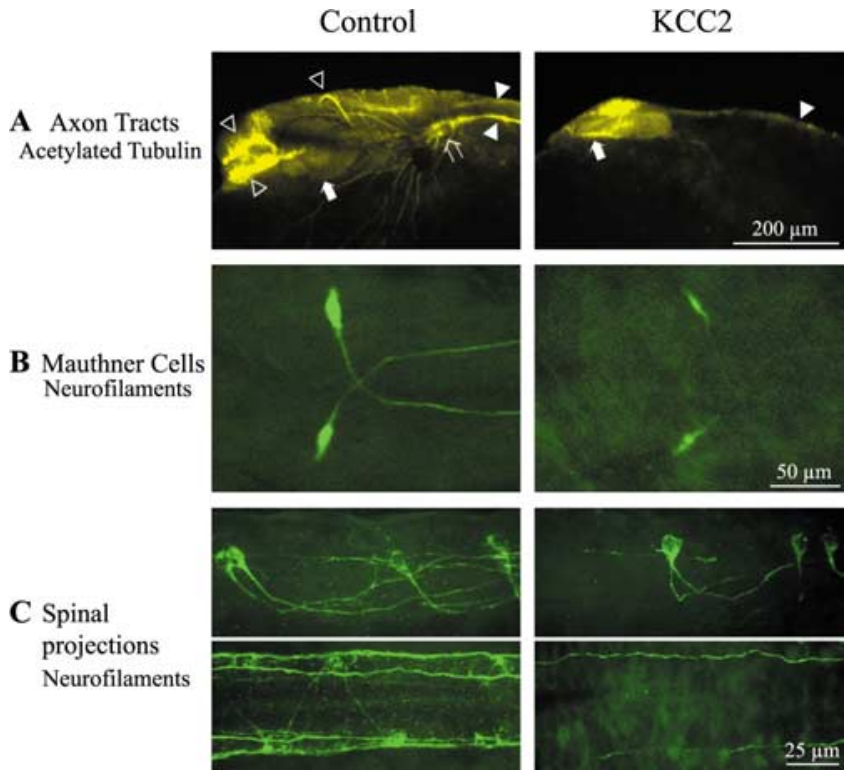

Figure 4. $\quad \mathrm{KCC} 2$ overexpression impairs the growth and maturation of brain and hindbrain structures. $A$, In control embryos, the acetylated tubulin staining highlights the postoptic, anterior, and posterior commissures (open arrowheads), the ventral and dorsal longitudinal tracts (filled arrowheads), the trigeminal ganglion (open arrow), and the pigmented optic cup forming the retina (filled arrow). In contrast, only the ventral longitudinal tract (filled arrowhead) and the optic vesicle (filled arrow) can be seen in KCC2-overexpressing embryos. $\boldsymbol{B}$, Top-down view of the large Mauthner cells, which are easily discernable in control embryos. When KCC2 is overexpressed, the Mauthner cells appear less well defined but their axons cross the midline and project caudally as in control embryos. C, The hindbrain reticulospinal neurons (side view) are present in KCC2 embryos. However, their axon projections to the spinal cord (top-down view) are much less elaborated in $\mathrm{KCC} 2$-overexpressing embryos.

subset of progenitor cells committed to a neuronal fate using an antibody to Dbx1 (Fig. 6D), a transcription factor expressed in the intermediate region of the neural tube involved in the specification of a subset of interneurons (Pierani et al., 1999). Similar to our observations when counting newly generated neurons, we found fewer Dbx1-labeled committed progenitor cells in the spinal cord of KCC2-expressing embryos (Table 1). These results indicate that early KCC2 expression impaired the birth of neurons without affecting the proliferation or death of their (uncommitted) progenitors.

The fact that spinal cord progenitors were not affected by KCC2 overexpression, despite a decrease in interneuron and motoneuron populations, suggested that proliferating cells could have switched fate and adopted a non-neuronal destiny. We therefore looked at glial cells, the alternate fate most likely adopted by the precursors (Park et al., 2004), expecting a dramatic increase in this population. Because astrocytes are found only in adult zebrafish (Lewis and Eisen, 2003), we focused on radial glia and oligodendrocyte precursors, both found in the embryo (Appel et al., 2001; Park et al., 2002). We did not observe an obvious increase in Zrf-1-labeled radial glial cells (Trevarrow et al., 1990) (Fig. 6E) or in oligodendrocyte precursors from PLP-GFP transgenic embryos (Yoshida and Macklin, 2005) (Fig. $6 F$ ) in embryos overexpressing KCC2. These observations indicated that the unaffected pool of progenitors did not adopt a glial fate and instead stalled before their last division.

\section{Discussion}

Ours is the first study wherein KCC2 overexpression was undertaken in vivo from the onset of development to reverse the chloride gradient throughout the embryo and thereby assess the
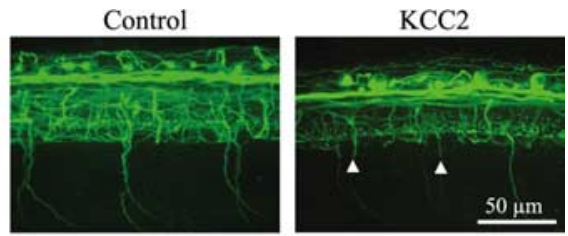

A Axonal Growth Acetylated Tubulin

B Neurogenesis $\mathrm{Hu}$
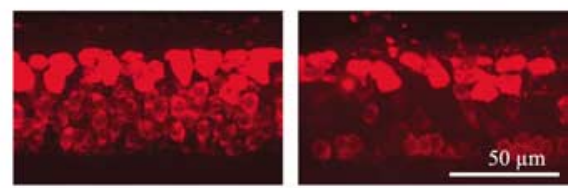

C Interneurons Pax 2
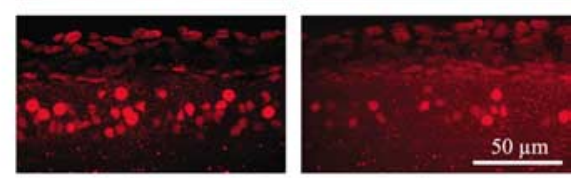

D Motoneurons HB9
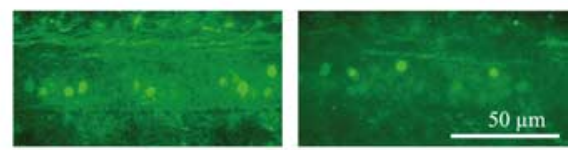

E Sensory Neurons
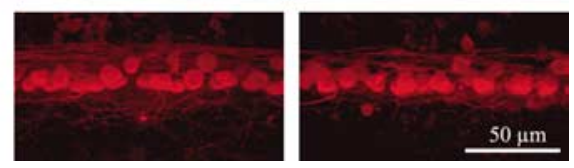

Figure 5. KCC2 overexpression perturbs axonal maturation and neurogenesis in the zebrafish spinal cord. A, Acetylated tubulin staining highlights the impairment in maturation and elaboration of axonal networks in the spinal cord during KCC2 overexpression. The arrowheads point to motoneuron axons that failed to extend completely out of the spinal cord. $\boldsymbol{B}$, Anti-Hu staining shows that KCC2-overexpressing embryos possess less newly born spinal neurons. When looking at specific neuronal types using post-differentiation markers, we observed decreased populations of interneurons expressing $\operatorname{Pax} 2(\boldsymbol{C})$ and motoneurons expressing HB9 (D). However, sensory neurons expressing NGN1 $(\boldsymbol{E})$ were not affected by the overexpression of KCC2.

Table 1. Cell counts in $100 \mu \mathrm{m}$ sections of the spinal cord

\begin{tabular}{lccc}
\hline & Control $(n)$ & KCC2 $(n)$ & $p$ value \\
\hline Hu & $107 \pm 3(16)$ & $67 \pm 4(9)$ & $<0.001$ \\
BrdU & $155 \pm 5(19)$ & $149 \pm 6(17)$ & 0.44 \\
PH3 & $23 \pm 1(8)$ & $21 \pm 1(3)$ & 0.15 \\
Dbx1 & $18 \pm 1(6)$ & $8 \pm 1(7)$ & $<0.001$ \\
Pax2 & $32 \pm 1(4)$ & $11 \pm 1(3)$ & $<0.001$ \\
HB9 & $17 \pm 2(19)$ & $9 \pm 2(7)$ & 0.04 \\
NGN1 & $15 \pm 1(28)$ & $17 \pm 1(9)$ & 0.11 \\
\hline
\end{tabular}

global impact on CNS development. Unlike previous studies, which used DNA transfection of cells in culture or DNA electroporation in vivo to achieve early KCC2 expression (Chudotvorova et al., 2005; Lee et al., 2005; Akerman and Cline, 2006; Liu et al., 2006; Cancedda et al., 2007), our technique of blastocyst mRNA injection allowed global expression of KCC2 and thus permitted the study of a reversal of the chloride gradient not in subsets of neurons but rather in populations of neurons interacting together. Zebrafish embryos precociously expressing KCC2 displayed immature morphological features and swimming behaviors. At a cellular level, we found reduced differentiation of spinal interneurons and motoneurons and consequently impaired axon growth throughout the CNS. Neural progenitors appeared not to degenerate or switch to a glial fate but rather stalled without further differentiating. Our study thus demonstrates the importance of the depolarizing chloride gradient in signaling the trophic effects of glycine and reveals the necessity for a delay in KCC2 expression. 


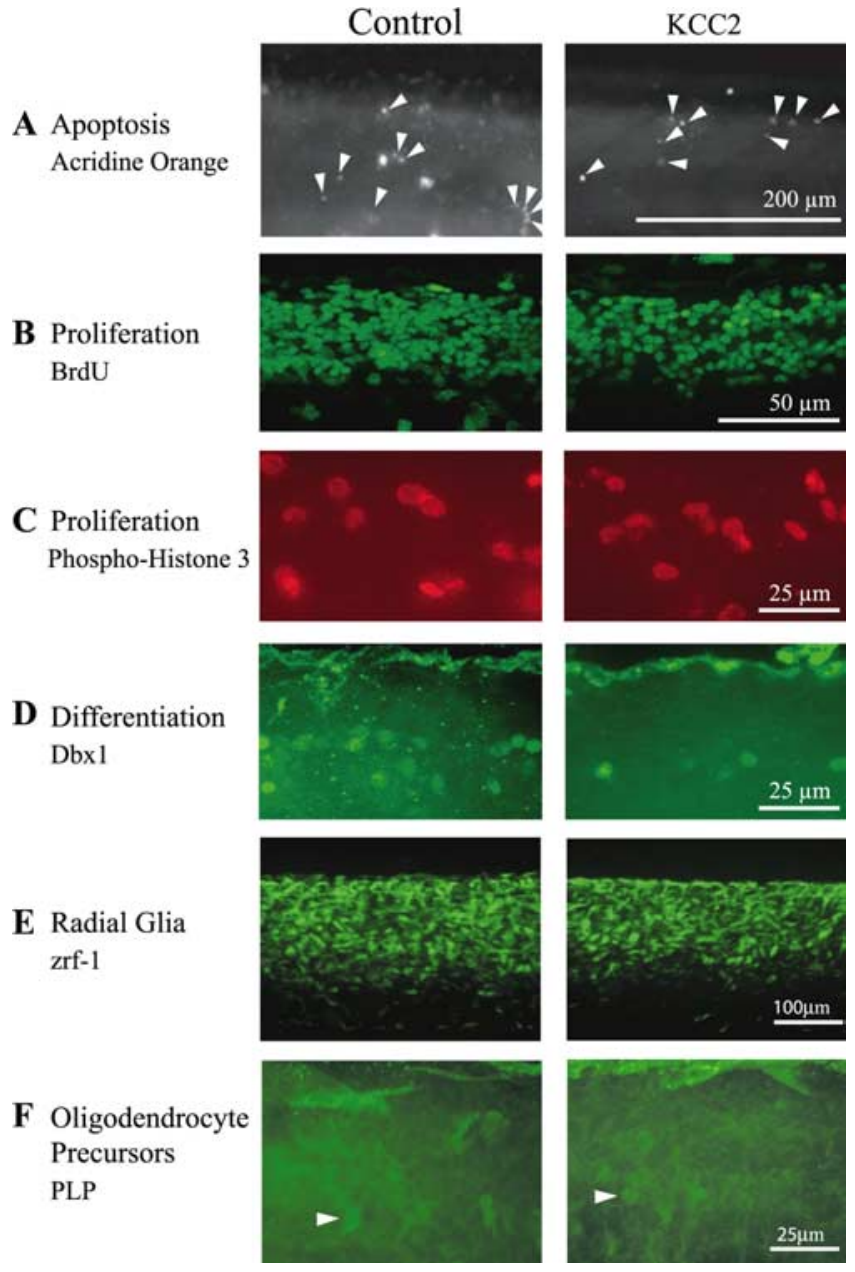

Figure 6. Effects of $\mathrm{KCC} 2$ overexpression on apoptosis proliferation and differentiation in the zebrafish spinal cord. $\boldsymbol{A}$, There is no obvious change in the levels of neurodegeneration during KCC2 overexpression because acridine orange-labeled apoptotic cells are not more abundant in the spinal cord of $\mathrm{KCC} 2$ embryos. Proliferation was not drastically modified by treatment because the number of BrdU-labeled cells $(\boldsymbol{B})$ and the number of phospho-histone-3-labeled cells $(\boldsymbol{C})$ is not significantly different between $\mathrm{KCC} 2$ and control embryos. In contrast, $(\boldsymbol{D})$ neural differentiation was compromised in $\mathrm{KCC} 2$ embryos because a subset of committed progenitors marked with Dbx1 was significantly fewer in the spinal cord of these embryos. E, Zrf-1 staining of radial glial cells in the spinal cord of control and KCC2 embryos $(n=26$ and $n=14$, respectively). $\boldsymbol{F}$, Fluorescent oligodendrocyte precursors in the spinal cord of PLP-GFP transgenics in non-injected zebrafish and zebrafish overexpressing $\mathrm{KCC}(n=9$ and $n=9$, respectively). The arrowheads point to such precursors, which were hard to distinguish at this stage of development in the spinal cord.

\section{Building a neuronal network before KCC2 expression}

The behavioral consequences of decreased neural differentiation during precocious expression of KCC2 in the zebrafish embryo were likely attributable to the inability to develop a completely functional locomotor neural network. The initial coiling behavior, which depends on electrical coupling and not on synaptic activity in the spinal cord (Saint-Amant and Drapeau, 2000), was observed and reflects some degree of functional neural output. However, the swimming activity in response to touch (Fig. 3B), which depends on integration of glycinergic and glutamatergic synaptic activity (Buss and Drapeau, 2001; Saint-Amant and Drapeau, 2001), failed to develop in embryos overexpressing KCC2. Our results thus indicate that, although some synapses were functional in KCC2 embryos, many aspects of synaptic integration failed to develop normally during reversal of the chloride gradient, which may underlie the loss of both glycine and glutamate synapses attributable to the loss of neurons, as observed previously during knockdown of chloride-permeant glycine receptors in zebrafish embryos (McDearmid et al., 2006).

In two other preparations in which the effects of KCC2 overexpression on synaptic homeostasis were examined, increased GABAergic activity without changes in glutamate activity was reported in cultured hippocampal neurons (Chudotvorova et al., 2005), whereas GABAergic activity increased while the normal developmental increase in glutamate activity was prevented in Xenopus embryos (Akerman and Cline, 2006). Although the different effects of KCC2 may be attributable to differences in the preparations studied, a consistent observation is that precocious overexpression of KCC2 affects synaptic homeostasis. Spontaneous recurrent activity within newly forming neural networks, be it attributable to synaptic or electrical coupling or both, is a hallmark of nervous system genesis. This activity is initially poor in informational content because the immature neurons of the forming networks cannot interpret and respond to activityencoded messages (Ben-Ari, 2002). Developing neurons thus appear to require discrete, periodic activity, not too much so as to cause excitotoxicity yet often enough to trigger developmental programs such as we observed for neurogenesis. In this perspective, the use of GABA and glycine as excitatory signals makes teleological sense because, unlike glutamate, they will not cause toxic levels of calcium entry attributable to their shunting effect (Ben-Ari, 2002; Owens and Kriegstein, 2002) and can thus cooperate in a homeostatic balance set in part by the depolarizing chloride gradient

\section{Normal proliferation with fewer neurons}

Using a variety of markers, we failed to find changes in cell proliferation in the spinal cord. The fact that the impaired birth of new neurons was not accompanied by changes in progenitor numbers during KCC2 overexpression is intriguing considering that GlyR $\alpha 2$ knockdown prevents exit from the cell cycle and significantly augments the progenitor population (McDearmid et al., 2006). It is possible that the mechanism regulating the exit of interneuron progenitors from the cell cycle relies on glycinergic signaling yet is less dependent on the chloride gradient and the associated excitation, unlike neuronal fate decisions. Supporting this possibility are results from a study on rat retinal progenitors using gain- and loss-of-function GlyR $\alpha 2$ (Young and Cepko, 2004), which highlighted the role of GlyR $\alpha 2$ in exit from the cell cycle and differentiation. In that study, the increase in rod progenitor proliferation detected during GlyR $\alpha 2$ overexpression was abolished when overexpressing channels with impaired ligand binding but was preserved when overexpressing GlyR $\alpha 2$ with mutated channel gating (Young and Cepko, 2004). These results indicate that chloride efflux through GlyR $\alpha 2$ channels and the depolarization thereby generated might not be the critical factors affecting proliferation of both rod photoreceptors in the rat retina and interneurons in the zebrafish spinal cord. The mechanisms in place might somehow rely more on the detection of glycine than on its depolarizing effect to arrest proliferation. In addition, other activity-dependent but chloride-independent mechanisms, such as the sodium current generated by reuptake of glycine by the sodium-dependent glycine transporter (Belachew et al., 2000), might be at work and remain to be investigated. It was recently reported that knock-out of GlyR $\alpha 2$ in mice failed to impact on CNS development (Young-Pearse et al., 2006). Although GABAergic signaling could obviate a requirement for glycinergic signaling in mouse embryos, GABAergic transmission is not established in the early zebrafish nervous system (Triller et al., 
1997; Buss and Drapeau, 2001). Thus, potential homeostatic effects of compensatory transmitter systems may not occur in zebrafish and could explain the strong impact of interfering with glycinergic transmission on neuronal development in this species.

\section{Glycine-induced depolarization and cell fate decisions}

The fewer Dbx1 cells observed in KCC2-expressing embryos suggest that, for some neurons, the activity of progenitors is a prerequisite for the adoption of a neural fate. The effect of GABA on interneuron differentiation has been shown previously in cultured hippocampal neurons, in which calcium elevations caused the expression of brain-derived neurotrophic factor (Berninger et al., 1995) known to induce a GABAergic fate (Marty et al., 1996). Similarly, in the adult hippocampus, GABA-induced excitation caused the expression of NeuroD, a positive transcriptional regulator of neuronal differentiation, thereby contributing to the increased number of mature neurons in the adult dentate gyrus (Ge et al., 2007).

Abolishing chloride depolarization had selective effects on spinal interneuron and motoneuron populations as sensory neurons were spared, indicating that a generalized defect such as overall retardation of development did not occur. The absence of spontaneous activity in embryonic zebrafish spinal sensory neurons (Drapeau et al., 1999; Saint-Amant et al., 2001) might mean that chloride-dependent activity does not influence the development of sensory neurons. In fact, at the larval stage, sensory Rohon-Beard neurons normally die, whereas mutations selectively suppressing Rohon-Beard neuron activity prolong their survival (Svoboda et al., 2001). Thus, sensory neuron development would appear to proceed by a different process. This may also occur in other animals in which sensory neurons as a rule are also more subject to excitatory than to inhibitory control. Furthermore, in some neurons, KCC2 expression is not correlated with a change in the chloride gradient, and this may be the same in sensory neurons, such as dorsal root ganglion neurons that lack KCC2 expression and retain a depolarizing chloride gradient into adulthood (Payne et al., 2003). These observations may indicate a less important developmental role for the chloride gradient in sensory neuron development.

\section{Fate of the progenitors}

In the absence of massive apoptosis, our data with glial markers suggest that progenitors did not differentiate into glia but rather stalled before their last division. Chloride cotransporters have been involved previously in disruptions of the cell cycle, because NKCC1 blockade with bumetanide in bronchial smooth muscles cell cultures inhibited the $\mathrm{G}_{1}-\mathrm{S}$ phase transition without facilitating apoptosis (Iwamoto et al., 2004). It is also possible that progenitors started committing to a neuronal fate by expressing ion channels and establishing a resting potential, but without expressing voltage-gated conductances or other postdifferentiation features such as neural-specific transcription factors or neurites. This hypothesis would explain why more neurons recorded in KCC2-expressing embryos had a normal resting potential without displaying spontaneous activity or synaptic innervation. A question for future studies, once more selective cell-type-specific markers become available, is whether there is a generalized dependence of neural differentiation on chloridedependent activity or whether there is an activity-dependent developmental program that generates specific sets of neurons. Although we cannot rule out a contribution of glia to neurogenesis, in the absence of a significant change in the glial population during KCC2 overexpression, we presume this contribution is relatively minor. Clearly, more investigation is required to understand the molecular and cellular changes underlying activitydependent neuronal differentiation. Nevertheless, our results demonstrate that depolarizing activity, through activation of chloride conducting ion channels, is essential at the earliest stage of CNS development to fully specify the neuronal populations and the consequent maturation and elaboration of axonal tracts to form neural circuits and networks. Thus, activity-dependent mechanisms are at play throughout the life of an animal.

\section{References}

Akerman CJ, Cline HT (2006) Depolarizing GABAergic conductances regulate the balance of excitation to inhibition in the developing retinotectal circuit in vivo. J Neurosci 26:5117-5130.

Appel B, Givan LA, Eisen JS (2001) Delta-Notch signalling and lateral inhibition in zebrafish spinal cord development. BMC Dev Biol 1:13.

Arber S, Han B, Mendelsohn M, Smith M, Jessell TM, Sockanathan S (1999) Requirement for the homeobox gene $\mathrm{Hb} 9$ in the consolidation of motor neuron identity. Neuron 23:659-674.

Belachew S, Malgrange B, Rigo JM, Rogister B, Leprince P, Hans G, Nguyen L, Moonen G (2000) Glycine triggers an intracellular calcium influx in oligodendrocyte progenitor cells which is mediated by the activation of both the ionotropic glycine receptor and $\mathrm{Na}^{+}$-dependent transporters. Eur J Neurosci 12:1924-1930.

Ben-Ari Y (2002) Excitatory actions of GABA during development: the nature of the nurture. Nat Rev Neurosci 3:728-739.

Berninger B, Marty S, Zafra F, da Penha Berzaghi M, Thoenen H, Lindholm D (1995) GABAergic stimulation switches from enhancing to repressing BDNF expression in rat hippocampal neurons during maturation in vitro. Development 121:2327-2335.

Blader P, Plessy C, Strahle U (2003) Multiple regulatory elements with spatially and temporally distinct activities control neurogenin 1 expression in primary neurons of the zebrafish embryo. Mech Dev 120:211-218.

Brustein E, Drapeau P (2005) Serotoninergic modulation of chloride homeostasis during maturation of the locomotor network in zebrafish. J Neurosci 25:10607-10616.

Brustein E, Chong M, Holmqvist B, Drapeau P (2003) Serotonin patterns locomotor network activity in the developing zebrafish by modulating quiescent periods. J Neurobiol 57:303-322.

Burrill JD, Moran L, Goulding MD, Saueressig H (1997) PAX2 is expressed in multiple spinal cord interneurons, including a population of EN1+ interneurons that require PAX6 for their development. Development 124:4493-4503.

Buss RR, Drapeau P (2001) Synaptic drive to motoneurons during fictive swimming in the developing zebrafish. J Neurophysiol 86:197-210.

Cancedda L, Fiumelli H, Chen K, Poo MM (2007) Excitatory GABA action is essential for morphological maturation of cortical neurons in vivo. J Neurosci 27:5224-5235.

Chudotvorova I, Ivanov A, Rama S, Hübner CA, Pellegrino C, Ben-Ari Y, Medina I (2005) Early expression of KCC2 in rat hippocampal cultures augments expression of functional GABA synapses. J Physiol (Lond) 566:671-679.

Drapeau P, Ali DW, Buss RR, Saint-Amant L (1999) In vivo recording from identifiable neurons of the locomotor network in the developing zebrafish. J Neurosci Methods 88:1-13.

Fiumelli H, Woodin MA (2007) Role of activity-dependent regulation of neuronal chloride homeostasis in development. Curr Opin Neurobiol 17:81-86

Furutani-Seiki M, Jiang YJ, Brand M, Heisenberg CP, Houart C, Beuchle D, van Eeden FJ, Granato M, Haffter P, Hammerschmidt M, Kane DA, Kelsh RN, Mullins MC, Odenthal J, Nusslein-Volhard C (1996) Neural degeneration mutants in the zebrafish, Danio rerio. Development 123:229-239.

Ge S, Pradhan DA, Ming GL, Song H (2007) GABA sets the tempo for activity-dependent adult neurogenesis. Trends Neurosci 30:1-8.

Iwamoto LM, Fujiwara N, Nakamura KT, Wada RK (2004) Na-K-2Cl cotransporter inhibition impairs human lung cellular proliferation. Am J Physiol Lung Cell Mol Physiol 287:L510-L514.

Kyrozis A, Reichling DB (1995) Perforated-patch recording with gramicidin avoids artifactual changes in intracellular chloride concentration. J Neurosci Methods 57:27-35. 
Lee H, Chen CX, Liu YJ, Aizenman E, Kandler K (2005) KCC2 expression in immature rat cortical neurons is sufficient to switch the polarity of GABA responses. Eur J Neurosci 21:2593-2599.

Lewis KE, Eisen JS (2003) From cells to circuits: development of the zebrafish spinal cord. Prog Neurobiol 69:419-449.

Liao D, Malinow R (1996) Deficiency in induction but not expression of LTP in hippocampal slices from young rats. Learn Mem 3:138-149.

Liu Z, Neff RA, Berg DK (2006) Sequential interplay of nicotinic and GABAergic signaling guides neuronal development. Science 314:1610-1613.

Marty S, Berninger B, Carroll P, Thoenen H (1996) GABAergic stimulation regulates the phenotype of hippocampal interneurons through the regulation of brain-derived neurotrophic factor. Neuron 16:565-570.

McDearmid JR, Liao M, Drapeau P (2006) Glycine receptors regulate interneuron differentiation during spinal network development. Proc Natl Acad Sci USA 103:9679-9684.

Owens DF, Kriegstein AR (2002) Is there more to GABA than synaptic inhibition? Nat Rev Neurosci 3:715-727.

Park HC, Mehta A, Richardson JS, Appel B (2002) olig2 is required for zebrafish primary motor neuron and oligodendrocyte development. Dev Biol 248:356-368.

Park HC, Shin J, Appel B (2004) Spatial and temporal regulation of ventral spinal cord precursor specification by Hedgehog signalling. Development 131:5959-5969.

Payne JA, Rivera C, Voipio J, Kaila K (2003) Cation-chloride cotransporters in neuronal communication, development and trauma. Trends Neurosci 26:199-206.

Pierani A, Brenner-Morton S, Chiang C, Jessell TM (1999) A sonic hedgehog-independent, retinoid-activated pathway of neurogenesis in the ventral spinal cord. Cell 97:903-915.

Plotkin MD, Snyder EY, Hebert SC, Delpire E (1997) Expression of the $\mathrm{Na}-\mathrm{K}-2 \mathrm{Cl}$ cotransporter is developmentally regulated in postnatal rat brains: a possible mechanism underlying GABA's excitatory role in immature brain. J Neurobiol 33:781-795.

Rivera C, Voipio J, Payne JA, Ruusuvuori E, Lahtinen H, Lamsa K, Pirvola U, Saarma M, Kaila K (1999) The $\mathrm{K}^{+} / \mathrm{Cl}^{-}$co-transporter KCC2 renders GABA hyperpolarizing during neuronal maturation. Nature 397:251-255.

Russell JM (2000) Sodium-potassium-chloride cotransport. Physiol Rev $80: 211-276$.
Saint-Amant L, Drapeau P (1998) Time course of the development of motor behaviors in the zebrafish embryo. J Neurobiol 37:622-632.

Saint-Amant L, Drapeau P (2000) Motoneuron activity patterns related to the earliest behavior of the zebrafish embryo. J Neurosci 20:3964-3972.

Saint-Amant L, Drapeau P (2001) Synchronization of an embryonic network of identified spinal interneurons solely by electrical coupling. Neuron 31:1035-1046.

Song L, Mercado A, Vazquez N, Xie Q, Desai R, Georges Jr AL, Gamba G, Mount DB (2002) Molecular, functional, and genomic characterization of human $\mathrm{KCC} 2$, the neuronal $\mathrm{K}-\mathrm{Cl}$ cotransporter. Brain Res Mol Brain Res 103:91-105.

Spitzer NC (2006) Electrical activity in early neuronal development. Nature 444:707-712.

Svoboda KR, Linares AE, Ribera AB (2001) Activity regulates programmed cell death of zebrafish Rohon-Beard neurons. Development 128:3511-3520.

Szabo A, Dalmau J, Manley G, Rosenfeld M, Wong E, Henson J, Posner JB, Furneaux HM (1991) HuD, a paraneoplastic encephalomyelitis antigen, contains RNA-binding domains and is homologous to Elav and Sexlethal. Cell 67:325-333.

Trevarrow B, Marks DL, Kimmel CB (1990) Organization of hindbrain segments in the zebrafish embryo. Neuron 4:669-679.

Triller A, Rostaing P, Korn H, Legendre P (1997) Morphofunctional evidence for mature synaptic contacts on the Mauthner cell of 52-hour-old zebrafish larvae. Neuroscience 80:133-145.

Westerfield M (1995) The zebrafish book: a guide for laboratory use of zebrafish (Brachydanio rerio). Eugene, OR: University of Oregon.

Wilson SW, Ross LS, Parrett T, Easter Jr SS (1990) The development of a simple scaffold of axon tracts in the brain of the embryonic zebrafish, Brachydanio rerio. Development 108:121-145.

Yamada J, Okabe A, Toyoda H, Kilb W, Luhmann HJ, Fukuda A (2004) $\mathrm{Cl}^{-}$ uptake promoting depolarizing GABA actions in immature rat neocortical neurones is mediated by NKCC1. J Physiol (Lond) 557:829-841.

Yoshida M, Macklin WB (2005) Oligodendrocyte development and myelination in GFP-transgenic zebrafish. J Neurosci Res 81:1-8.

Young TL, Cepko CL (2004) A role for ligand-gated ion channels in rod photoreceptor development. Neuron 41:867-879.

Young-Pearse TL, Ivic L, Kriegstein AR, Cepko CL (2006) Characterization of mice with targeted deletion of glycine receptor alpha 2. Mol Cell Biol 26:5728-5734. 